February. The overall pass rate is certainly lower for overseas candidates than for British candidates (in Autumn $1984,25 \%$ versus $66 \%$ in the Preliminary Test, and $34 \%$ versus $68 \%$ in the Membership Examination). These results, which are fairly typical, are discouraging, but not quite as bad as the six to one chance of failure which $\mathrm{Dr}$ Onyango has calculated.

As reported at the Forum, I have witnessed no discrimination against overseas doctors in the clinical and oral examinations. Although most examiners now use numbers and not names when examining candidates, this is not an efficient way of concealing the origins of most doctors. Psychiatrists, like everyone else, can make deductions from colour, shape, accent and habit. The papers and MCQs are marked anonymously and the results of the examination depend entirely on the marks obtained in its various sections.

I hope this will give some reassurance to Dr Onyango. For myself, I would like to be reassured that unsuccessful candidates take advantage of the opportunity of obtaining some feedback on their performance which can now be sent directly to themselves, but should wherever possible be discussed with their tutor. The College does have regulations to ensure that trainees in their later attempts have received one year's approved training between attempts, but the decision whether to enter for a further attempt must ultimately rest with the trainee and not with the tutor, who can only advise on this matter.

[See pages 59-60 of this issue for the report of the Trainees' Forum, 'Why Do Overseas Trainees Fail?']

\section{ECT on an out-patient basis}

\section{DeAr SIRS}

Electroconvulsive therapy is a valuable and effective treatment for major depressive disorder (DSM-III) and the depressed phase of manic-depressive psychosis (ICD-9). It may also be the treatment of choice for the acute schizoaffective states following childbirth, restoring the woman to health and contact with her baby more quickly than drug treatment. I have also seen many patients with prolonged depressive states following childbirth who have been dubbed 'neurotic' or 'inadequate mothers' by other psychiatrists, who have returned to normal effective functioning following a few ECT treatments given on an out-patient basis. It appears that many psychiatrists do not advise ECT on an out-patient basis and so those patients with milder, but nonetheless ECT-responsive depressive states, must either be admitted to a psychiatric hospital or else denied the treatment.

One of the factors which influences many psychiatrists against the prescription of ECT on an out-patient basis is the possibility of misadventure and of subsequent disaster or litigation. Although in the twenty years that I have been prescribing ECT on an out-patient basis I have not met with disaster, I recognise that luck was on my side.

The ECT Unit Staff (Sister Pullman and the staff nurses) and I recently decided that an information leaflet for patients and their relatives would be of value. I took advice from Dr Wright, the day care consultant anaesthetist at this hospital, and I thought it would be a precaution to submit our proposed leaffet to the Medical Protection Society. Both Dr Wright and Dr J. J. Bradley (replying on behalf of the MPS) made helpful suggestions and ammendments. Dr Bradley commented that he wishes such a document had been in existence when he worked as a senior registrar in London in the 1960 s for he recalled a London Transport driver who addmitted that he left the ECT clinic and took charge of his bus on the same day!

Since the document has come into use at St James's Hospital, several colleagues in other hospitals in Yorkshire have adopted it. I am grateful to the Editors of the Bulletin for reproducing the Information Leaflet since other psychiatrists may also wish to introduce it or a similar document. The form of the document reprinted here carries no copyright and may be adapted for local use.

R.P. SNATTH

\section{St James's University Hospital}

Leeds

\section{Information Leaflet}

ECT is a very effoctive treatment and has been used for over 50 years. The nature and purpose of the treatment will have been explained to you when you signed the form of your consent to treatment.

If you are having the treatment as an out-patient, there are some rules which must be followed because you will have a brief anaesthetic which will be given by injection into a vein in your arm.

1. You must not have anything to eat or drink after midnight on the day before your treatment.

You may have a mouth rinse or clean your teeth in the morning, but avoid swallowing much water.

If you are taking tablets in the morning, don't take them on the morning of your treatment; bring them with you and give them to the nurse who will give them to you with your cup of tea after the treatment.

2. If you develop a severe head cold during the course of your treatment you may not be able to have an anaesthetic on a day when the cold is very bad: so ask someone to telephone and you will be told on which day you should next come. The telephone number is:...

3. You must not drive a car or any motor vehicle on the day on which you have a treatment.

4. Ambulance transport can be arranged, but it would be best if a relative or friend can drive you to the hospital and take you home again. You should arrive before $9.15 \mathrm{am}$ and will be ready to go home again around $11.30 \mathrm{am}$.

You should not travel unaccompanied.

5. You should not return to an empty house. Therefore if transport is arranged for you, please arrange for a relative or neighbour to be at your home when you return.

ECT is not an unpleasant treatment although you may have a slight 'muzzy' feeling or headache after you wake up from the anaesthetic; this generally passes off after you have drunk a cup of tea which the nurse will bring you.

The treatment does not have an immediate effect so don't be worried if you do not feel better after the first few treatments. If you wish to discuss your progress with your doctor before any of your treatment sessions, let the nurse know when you arrive. 